\title{
Modelling of Interconnected Critical Infrastructure Systems Using Complex Network Theory
}

DOI:

10.1109/TSG.2017.2665646

\section{Document Version}

Accepted author manuscript

Link to publication record in Manchester Research Explorer

\section{Citation for published version (APA):}

Milanovic, J. V., \& Zhu, W. (2017). Modelling of Interconnected Critical Infrastructure Systems Using Complex Network Theory. IEEE Transactions on Smart Grid, (99). https://doi.org/10.1109/TSG.2017.2665646

\section{Published in:}

IEEE Transactions on Smart Grid

\section{Citing this paper}

Please note that where the full-text provided on Manchester Research Explorer is the Author Accepted Manuscript or Proof version this may differ from the final Published version. If citing, it is advised that you check and use the publisher's definitive version.

\section{General rights}

Copyright and moral rights for the publications made accessible in the Research Explorer are retained by the authors and/or other copyright owners and it is a condition of accessing publications that users recognise and abide by the legal requirements associated with these rights.

\section{Takedown policy}

If you believe that this document breaches copyright please refer to the University of Manchester's Takedown Procedures [http://man.ac.uk/04Y6Bo] or contact uml.scholarlycommunications@manchester.ac.uk providing relevant details, so we can investigate your claim.

\section{OPEN ACCESS}




\title{
Modelling of Interconnected Critical Infrastructure Systems Using Complex Network Theory
}

\author{
Jovica V. Milanović, Fellow, IEEE, and Wentao Zhu, Student Member, IEEE
}

\begin{abstract}
The paper reviews several Complex Network Theory (CNT) based methodologies to model and analyze interconnected networks. To address the deficiencies reported in past studies, an advanced model of coupled Electric Power System (EPS) and Information and Communication Technology (ICT) is developed. The new model is designed as a three-dimensional graph with edges and nodes, and it highlights the interaction and interdependency of both EPS and ICT. The criticality of each component within the interconnected system is investigated by analyzing the efficiency-weighted node degree of each node symbolizing the components. The model has been tested on an ICT supported 14-Bus distribution network under three different loading conditions. Results show that the coupled infrastructure complies with the typical characteristics of scale-free network [1]. It is also shown the ICT system, although smaller in scale, contains more critical hubs than the power distribution network. The results of the proposed approach can be used as a reference to analyze the criticality in other coupled infrastructure by incorporating the internal characteristics of coupled systems.
\end{abstract}

Index Terms-Critical infrastructure systems, Power systems, ICT, Complex networks

\section{INTRODUCTION}

$\mathrm{E}$ LECTRIC Power Systems (EPS) of the future, in addition to ever increasing complexity in their own right due to proliferation of intermittent and stochastic power electronics interface connected renewable energy sources (RES), both generation and storage technologies, and new types of temporal and spatial varying loads, are expected to incorporate very large number of different Information and Communication Technologies (ICTs), that will enable full observability, controllability and flexibility of operation of the system. Various aspects of operation of both of these systems, EPS and ICT system have been extensively studied in the past as standalone systems and it has been proven that their secure operation is essential for society at large. The influence on society in case of their failure to operate as intended is huge and they are commonly referred to as critical infrastructure system together with gas, water, transport and heat networks. Each of the critical infrastructure systems mentioned above

This work was partly funded by the Research Councils UK, through the HubNet consortium (grant number: EP/I013636/1).

J.V. Milanović and W. Zhu are with the School of Electrical and Electronic Engineering, The University of Manchester, PO Box 88, Manchester, M60 1QD, UK. (email: milanovic@ manchester.ac.uk). has been traditionally studied independently and various methods and tools have been developed for their analysis. The societal push towards sustainable and affordable energy future and ever increasing interdependences of these systems are calling for new tools and methodologies for the analysis of coupled critical infrastructure systems, i.e., system-of-systems.

In case of coupled infrastructure system, a failure initiated within one system could cause cascading effects within the whole system-of-systems. For example, the US 2003 blackout [2] was initiated to a large extent by the failure that initially occurred in the ICT system. The increasing number of cyberattacks in particular put the ICT dependent systems (whether they are electric, gas or water systems [3]) under threat. The malicious attacks on ICT systems could be more effective than to physically attack the actual ICT controlled infrastructure [4] as demonstrated in [5], where the computer worm Stuxnet specifically targeted industrial control systems used in energy infrastructure. This emphasizes the importance of the security and reliability of coupled critical infrastructure systems. In order to identify the vulnerabilities of coupled systems, dependencies of only physical layers are often not sufficient to solve the problem. Four types of interdependencies of Critical Infrastructures have been identified in [6] as: i) Physical - a physical reliance on material flow from one infrastructure to another, e.g., power grids supply electric power to machine tool plants to run the illuminating systems and control systems; ii) Cyber - a reliance on information transfer between infrastructures. Also known as "Informational Interdependency", e.g., SCADA system monitors and controls power system components; iii) Geographic - a local environmental event affects components across multiple infrastructures due to physical proximity. Also known as "Geospatial Interdependency", e.g., all assets and equipment within one area get affected in an event of fire or flood; iv) Logical - a dependency that exists between infrastructures that does not fall into one of the above categories. Also known as "Policy/Procedural Interdependency”, e.g., Distribution System Operators (DSOs) started re-energization of customers without proper coordination with their Transmission System Operators (TSOs), resulting in a worsened situation to restore normal system conditions in a controllable way [7]. Additionally, a fifth type of interdependency called 'Social' is introduced in [8], which describes the impact of human behaviors on infrastructures, e.g., civilian procession blocks public transport 
system. There have been a reasonably small number of studies, compared to number of studies addressing individual systems in the past, which addressed coupled infrastructure systems, mostly two interconnected systems [9-13]. However, this research is still at very early stage.

Some of the methods used to model and analyze interconnected infrastructure systems include Input-Output inoperability/interdependence models, Agent Based Models, Fuzzy Dynamic Input Output Inoperability Model, Bayesian Networks, Petri Networks, Fault Trees, Failure Mode and Effect Analysis and Monte Carlo or Lifetime Assessment [14, 15]. Nevertheless, they did not provide yet an appropriate tool to study and understand the structure of coupled infrastructures and the results are sometimes too abstract to be understood. The critical analyses of various methods including their advantages and disadvantages are presented in [16]. It is shown there that the Complex Network Theory, developed from Graph Theory, provides an attractive tool to reveal the hidden interdependencies of large complex systems with nontrivial topologies [17]. This approach facilitates the development of cascading phenomena studies among interconnected systems. Cyber-physical systems have been studied in the past using graph theory. However, the graphical representations were mainly related to linear time-invariant descriptor system studies which focus on systems' states and the considered interconnections between two systems were all cyber connections [18]. However, the cyber components often, if not always, receive the power to operate directly from power systems, thus creating a strong interdependency between the two networks [19].

To begin with, this paper introduces three conventional centrality measuring indices, namely node degree, betweenness and closeness [20] based on Complex Network Theory, to analyze coupled EPS and ICT networks and to identify the critical hubs/buses that could initiate cascading failures in the interconnected network. The indices (Node degree, Betweenness Centrality, and Efficiency) introduced in [21] are modified to achieve proper mathematical representation of nodes' interdependencies by introducing a hybrid bidirectional and unidirectional complex network model to relate the real networks' operating conditions. In addition, the paper defined an Efficiency-weighted Node Degree (EWND), based on Node Degree and Efficiency, to globally assess the criticality and vulnerability of each node.

The proposed method differs from the past related studies in mainly three aspects: i) the cyber-physical network is studied for the first time as an integrated three-dimensional interconnected system; ii) the impact of different directions of information/power flows are incorporated in the proposed method and are discussed in relation to system vulnerability; iii) a new index is proposed based on conventional Complex Network indices to better describe the relationship between interconnected systems. Furthermore, the case study based on interconnected "smart distribution network" and communication network, facilitates the analysis and expansion design of an integrated Smart Grid model.

\section{Methodology: COMPleX Network TheORY}

\section{A. Graphical Representation}

A graph representation is the first step needed to study the interconnected network using Complex Network Theory (CNT) [22]. A graph $G$ is composed of two components, nodes (vertices) and edges (links). It is an ordered pair of sets (V, E), in which $V \equiv\left\{\mathrm{v}_{1}, \mathrm{v}_{2}, \mathrm{v}_{3}, \ldots, \mathrm{v}_{\mathrm{a}}\right\}$ (a is the number of nodes), and $E \equiv\left\{\mathrm{e}_{1}, \mathrm{e}_{2}, \mathrm{e}_{3}, \ldots, \mathrm{e}_{\mathrm{b}}\right\}$ (b is the number of edges). In the cyber-physical network, nodes represent the power buses and the ICT routers/multiplexers, and edges are the connections between a pair of nodes. They represent relations or dependencies, which are classified into the following four categories: i) Category 1: The power flow from an electric node to another electrical node; ii) Category 2: The information flow from an ICT node to another ICT node; iii) Category 3: The electric energy supply from an electric node to an ICT node; iv) Category 4: The sensory data/control command from/to an electric node to/from an ICT node.

1) Bidirectional Graph: The topology of an interconnected network can be simply presented in a bidirectional graph. Each edge is connected with two nodes. Nodes and edges are divided into two types $V_{e} \equiv\left\{\mathrm{v}_{\mathrm{e} 1}, \mathrm{v}_{\mathrm{e} 2}, \mathrm{v}_{\mathrm{e} 3}, \ldots, \mathrm{v}_{\mathrm{ep}}\right\}$ and $V_{c} \equiv\left\{\mathrm{v}_{\mathrm{c} 1}, \mathrm{v}_{\mathrm{c} 2}, \mathrm{v}_{\mathrm{c} 3}, \ldots, \mathrm{v}_{\mathrm{cq}}\right\}$ ( $p, q$ are the number of electrical and cyber nodes respectively), and $E_{e} \equiv\left\{\mathrm{e}_{\mathrm{e} 1}, \mathrm{e}_{\mathrm{e} 2}, \mathrm{e}_{\mathrm{e} 3}, \ldots, \mathrm{e}_{\mathrm{es}}\right\}$ and $E_{c} \equiv\left\{\mathrm{e}_{\mathrm{c} 1}, \mathrm{e}_{\mathrm{c} 2}, \mathrm{e}_{\mathrm{c} 3}, \ldots, \mathrm{e}_{\mathrm{ct}}\right\}$ ( $s, t$ are the number of electrical and cyber edges respectively), to represent the sets of electrical and cyber vertices/edges respectively. Fig. 1 shows an interconnected EPS-ICT bidirectional graph for demonstration purpose. The connections between two nodes represent a physical or cyber dependency. The effectiveness of using bidirectional graph to study the interdependent networks is discussed in [23].

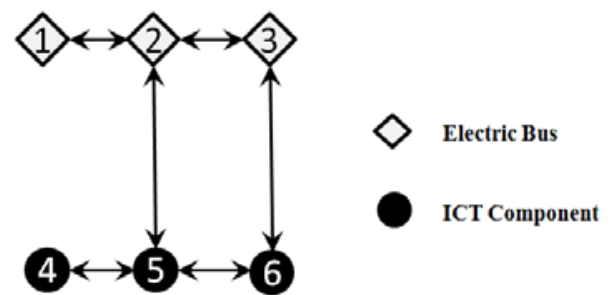

Fig. 1. Bidirectional model demonstration graph

2) Unidirectional Graph: In the case of EPS-ICT network, however, the interdependencies are not purely bidirectional. Therefore, the unidirectional model is developed. For unidirectional graphs, directions are assigned to each edge, and interactions between two systems are modelled independently as a physical dependency and a cyber dependency, as shown in Fig. 2.

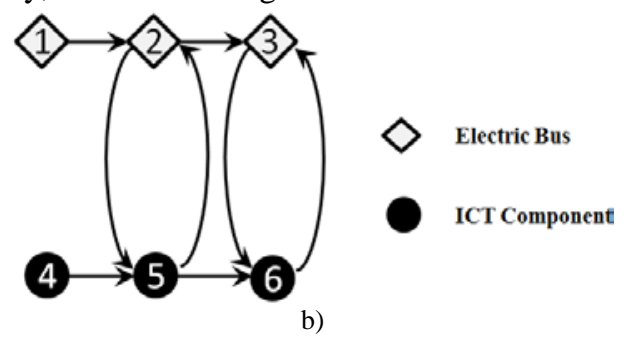

Fig. 2. Unidirectional graph demonstration graph 
3) Three-dimensional Interconnected Model: Cascading failure analyses, such as introduced in [23], have limitations of modeling cascading failures by describing interdependencies between two nodes either as unidirectional edge or bidirectional edge (e.g., the loss of power supply to an ICT router will not cause a power plant to collapse, however, the loss/failure of a power bus might result in the failure of the ICT router which may cause further issues.). A threedimensional model to study interdependent network was introduced in [24]. However, the interactions are only modelled as unidirectional edges, which cannot reflect the real network behavior of the coupled system. Inspired by the multi-layered infrastructure interdependencies model presented in [25], the three-dimensional model shown in Fig. 3 is developed to integrate the previous bidirectional and unidirectional models, providing more details of the coupled system's engineering structure and allowing for more flexibility.

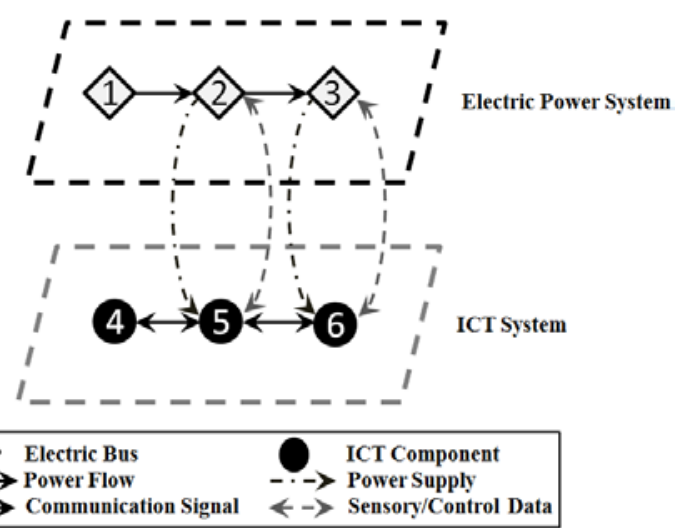

Fig. 3. Three-dimensional model demonstration graph

\section{B. Complex-valued adjacency matrix}

The graph representation of the interconnected system can be transformed into a complex-valued $n \times n$ adjacency matrix $\mathcal{A}$ [26]. Each row and column represents a node. Nodes' connections are described by matrix element $a_{h j}$, which represents the topological relationship between node $h$ and node $j$. The entry $a_{h j}$ (or connection $(h, j)$ ) is crucial in clarifying the interdependencies within the coupled system.

The equation (1) defines the rule of the formation of the bidirectional graph. The interdependencies within the unidirectional graph are described by (2) and (3). Equation (2) defines entries of matrix whose columns interpret nodes' dependencies to the system, and the rows, defined by (3), represent nodes' importance to the system. A threedimensional graph is represented by two matrices, equation (4) representing the electric connections and equation (5) representing the ICT connections. They describe how the electric and ICT matrices should be constituted respectively.

$$
\begin{aligned}
& \mathbf{a}_{\mathbf{h j}}= \begin{cases}1 & \text { if }(h, j) \text { belongs to category } 1 \\
i & \text { if }(h, j) \text { belongs to category } 2 \\
1+i & \text { if }(h, j) \text { belongs to category } 3 \text { or } 4 \\
0 & \text { otherwise }\end{cases} \\
& \mathbf{a}_{\mathbf{h j}}^{\mathbf{i n}}= \begin{cases}1 & \text { if }(h, j) \text { belongs to category } 1 \text { or } 3 \\
i & \text { if }(h, j) \text { belongs to category } 2 \text { or } 4 \\
0 & \text { otherwise }\end{cases}
\end{aligned}
$$

$$
\begin{aligned}
& \mathbf{a}_{\mathbf{h j}}^{\mathbf{o u t}}= \begin{cases}1 & \text { if }(h, j) \text { belongs to category } 1 \text { or } 4 \\
i & \text { if }(h, j) \text { belongs to category } 2 \text { or } 3 \\
0 & \text { otherwise }\end{cases} \\
& \mathbf{a}_{\mathbf{h j}}^{\mathbf{e}}= \begin{cases}1 & \text { if }(h, j) \text { belongs to category } 1 \\
1+i & \text { if }(h, j) \text { belongs to category } 3 \\
0 & \text { otherwise }\end{cases} \\
& \mathbf{a}_{\mathbf{h j}}^{\mathbf{c}}= \begin{cases}i & \text { if }(h, j) \text { belongs to category } 2 \\
1+i & \text { if }(h, j) \text { belongs to category } 4 \\
0 & \text { otherwise }\end{cases}
\end{aligned}
$$

As a result, the adjacency matrix for bidirectional model demonstration graph in Fig. 1 is defined in (6) as an illustrative example.

$$
A=\left[\begin{array}{cccccc}
0 & 1 & 0 & 0 & 0 & 0 \\
1 & 0 & 1 & 0 & 1+i & 0 \\
0 & 1 & 0 & 0 & 0 & 1+i \\
0 & 0 & 0 & 0 & i & 0 \\
0 & 1+i & 0 & i & 0 & i \\
0 & 0 & 1+i & 0 & i & 0
\end{array}\right]
$$

\section{Complex-valued Node Degree}

The importance of a node $h$ (representing an EPS bus or an ICT router/multiplexer) can be quantified by the number of nodes that it is incident with, i.e. the Node Degree (ND) $k_{h}$ [20]. Network robustness studies have suggested that networks become less resilient to attacks specifically focusing on high degree nodes [27] and have validated this conclusion on the available US power grid data [28-29]. These studies highlight the impact of the most highly connected nodes on the robustness of a network and their vulnerability to cascading failures in two interdependent systems [30].

In order to measure the influence of a particular node not only within its own infrastructure but also on the other, the ND is divided into two components calculated using the adjacency matrix $\mathcal{A}: k_{e h}$, the electrical ND, and $k_{c h}$, the ICT ND. A node has higher impact on the electric system if $k_{e h}>$ $k_{c h}$. For bidirectional graphs, the relationship of EPS and ICT ND to total node degree $k_{i}$, is shown in (7) [16].

$$
k_{h}=\sum_{j \in V} a_{h j}=k_{e h}+i \cdot k_{c h}
$$

For unidirectional graph, the ND of a node $h$ in corresponding network contains two components, in-degree $k_{h}^{i n}$, which is calculated in terms of the ingoing links, and outdegree $k_{h}^{\text {out }}$, calculated in terms of the outgoing links, as shown in (8) and (9).

$$
\begin{gathered}
k_{h}^{\text {in }}=\sum_{j \in V} a_{j h}=k_{e h}^{\text {in }}+i \cdot k_{c h}^{\text {in }} \\
k_{h}^{\text {out }}=\sum_{j \in V} a_{h j}=k_{\text {en }}^{\text {out }}+i \cdot k_{c h}^{\text {out }}
\end{gathered}
$$

The complex-valued ND of a node $i$ in the threedimensional model also contains two components, $k_{h}^{i n}$ and $k_{h}^{\text {out }}$. A bidirectional information edge gives the nodes connected with it both an in-degree and an out-degree. The results of complex-valued ND for unidirectional model demonstration graph in Fig. 2 are presented in Table I. 
TABLE I NoDe Degree - Unidirecitonal Model Demonstration Graph

\begin{tabular}{|c|c|c|c|c|c|c|}
\hline Node & 1 & 2 & 3 & 4 & 5 & 6 \\
\hline$k_{h}^{\text {in }}$ & 0 & $1+\mathrm{i}$ & $1+\mathrm{i}$ & 0 & $1+\mathrm{i}$ & $1+\mathrm{i}$ \\
\hline$k_{h}^{\text {out }}$ & 1 & $1+\mathrm{i}$ & $\mathrm{i}$ & $\mathrm{i}$ & $1+\mathrm{i}$ & 1 \\
\hline
\end{tabular}

\section{Path Length and Geodesics}

The path length is the physical distance from one node to another. In a binary graph, it is the number of edges existing between two nodes. The geodesic (the shortest path) is the minimum number of edges that need to be passed through from one node to another. Many algorithms have been developed to find the geodesics in a graph. The Dijkstra's algorithm [31] is chosen and applied to analyze the graph properties in this paper, which serves as the prerequisite of calculating the following network indices including Betweenness Centrality, Efficiency and Efficiency-weighted Node Degree.

\section{E. Betweenness Centrality (BC)}

The ND measures the centrality of a node within the network based on the number of ties each node has. It has some deficiencies, however, in identifying interconnected nodes. For instance, in Fig. 4, nodes 4 and 6 have the highest $\mathrm{ND}$ in the interconnected system, nevertheless, the interconnection node 5, which bridges the two systems, is obviously more topologically centralized than node 4 and 6 . To quantify centrality from another angle, [32] for the first time defined betweenness centrality of a node in a network based on the intermediary role that the node has between other nodes. Subsequently, the research on cascading failures using Complex Network Theory revealed that a power network is less resilient to the removal of critical node with the largest betweenness [33].

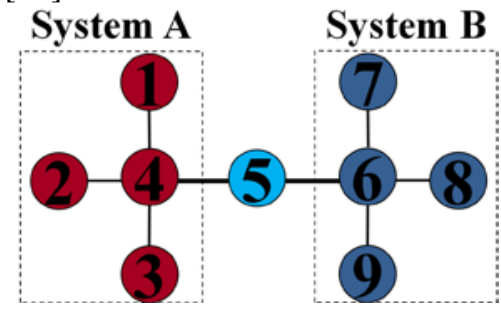

Fig. 4. Betweenness centrality demonstration graph

Betweenness Centrality of a node (or an edge) $x$ is calculated by (10) based on the number of shortest paths between any pair of nodes in the network passing through node (or edge) $x$. The shortest path (geodesic) between node $h$ and $j$, is the minimum number of edges from node $h$ to node $j$.

$$
C(x)=\sum_{h, j \in V, h \neq j} \frac{\sigma_{h j}(x)}{\sigma_{h j}}
$$

where $\sigma_{h j}$ is the total number of shortest paths existing between node $h$ and node $j$, and $\sigma_{h j}(x)$ is the number of shortest paths between node $h$ and node $j$ that pass through node (or edge) $x$.

In order to highlight the interdependencies between the electric power system and the ICT system, and to study their interplays, Betweenness Centrality is divided into two categories, the electric Betweenness Centrality $\mathrm{C}_{\mathrm{e}}(\mathrm{x})$ and the ICT Betweenness Centrality $\mathrm{C}_{\mathrm{c}}(\mathrm{x})$. They are treated as two- layer systems and are studied independently, as shown in (11) and (12). A global Betweenness Centrality $C_{\text {global }}(n)$ is introduced in (13) to create a collective index for quantifying the impact a node (or an edge) has on both EPS and ICT systems.

$$
\begin{gathered}
C_{e}(x)=\sum_{h, j \in V, h \neq j} \frac{\sigma_{e, h j}(x)}{\sigma_{e, h j}} \\
C_{c}(x)=\sum_{h, j \in V, h \neq j} \frac{\sigma_{c, h j}(x)}{\sigma_{c, h j}} \\
C_{\text {global }}(x)=\sqrt{C_{e}^{2}(x)+C_{c}^{2}(x)}
\end{gathered}
$$

where $\sigma_{e, h j}(x)$ is the number of shortest paths between electric nodes $h$ and $j$ that pass through node (or edge) $x$, and $\sigma_{c, h j}(x)$ is the number of shortest paths between ICT nodes $h$ and $j$ that pass through node (or edge) $x$.

The results of node BC analysis of the three-dimensional model demonstration graph in Fig. 3 are presented in Table II. It can be seen that node 2 and node 5 are the most critical nodes within EPS and ICT network.

TABLE II Node BC Results - Three-Layer Model Demonstration Graph

\begin{tabular}{|c|c|c|c|c|c|c|}
\hline Node & 1 & 2 & 3 & 4 & 5 & 6 \\
\hline$c_{e}$ & 0 & 3 & 2 & 0 & 0 & 0 \\
\hline$c_{c}$ & 0 & 0 & 0 & 0 & 10 & 6 \\
\hline$c_{\text {global }}$ & 0 & 3 & 2 & 0 & 10 & 6 \\
\hline
\end{tabular}

\section{F. Efficiency}

Efficiency (14) measures how efficiently the information within a system is exchanged between nodes [34]. It shows the effectiveness in studying standalone systems such as power systems [35]. The methodology to analyze efficiency of interconnected systems, however, is still under development.

$$
E=\frac{1}{m(m-1)} \sum_{h, j \in V, h \neq j} \frac{1}{d_{h j}}
$$

where $d_{h j}$ represents the length of shortest path between nodes $h$ and $j$ and $m$ is the number of nodes within the system.

The importance of a node (or an edge) is assessed based on the drop of global efficiency $\Delta \mathrm{E}(\mathrm{Y})$, given by (15), after removing that node (edge) from the system. The removal of a node or an edge represents the complete failure of that system component and power system backup batteries and generations and ICT redundancy channels are not accounted. The larger the efficiency drop, the more critical the node or the edge is in that system.

$$
\Delta \mathrm{E}(\mathrm{Y})=\frac{E(Y)-E(Y-1)}{E(Y)}
$$

where $E(Y)$ is the system normal state efficiency (or unperturbed efficiency) and $\mathrm{E}(\mathrm{Y}-1)$ is the system efficiency after the removal of a node or an edge.

To highlight the topological importance of a node (edge) and the role of that particular node (edge) plays in the interconnected system, the efficiency of the interconnected EPS-ICT system is divided here into electrical efficiency (16) and ICT efficiency (17), where $n_{e}$ and $n_{c}$ represent the number of electric nodes and ICT nodes respectively.

$$
\mathrm{E}_{e}=\frac{1}{n_{e} n_{c}} \sum_{h \in V_{e}, j \in V_{c}, h \neq j} \frac{1}{d_{h j}}
$$




$$
\mathrm{E}_{c}=\frac{1}{n_{e} n_{c}} \sum_{h \in V_{c}, j \in V_{e}, h \neq j} \frac{1}{d_{h j}}
$$

The results of the three-dimensional model demonstration graph's (in Fig. 3) EPS and ICT system efficiencies after the removal of each node are presented in Table III. Node 2 and node 5 are identified as the most critical nodes within EPS and ICT network respectively.

TABLE III Node EfFiciency - ThreE-LAYER Model Demonstration Graph

\begin{tabular}{|c|c|c|c|c|c|c|c|}
\hline Node & Normal & 1 & 2 & 3 & 4 & 5 & 6 \\
\hline $\mathrm{E}_{e}$ & 0.37 & 0.28 & 0.11 & 0.17 & 0.37 & 0.20 & 0.17 \\
\hline $\mathrm{E}_{c}$ & 0.93 & 0.93 & 0.41 & 0.44 & 0.74 & 0.22 & 0.33 \\
\hline
\end{tabular}

\section{G. Efficiency-weighted Node Degree (EWND)}

The canonical measures of centrality show some effectiveness in identifying critical hubs, but they all have intrinsic limitations in studying the interconnected system. Node Degree measures the importance of a node $i$ based on the number of connections it has, but underestimates the importance of central nodes with few connections. Betweenness-based measures of centrality solved this problem but they cannot distinguish the roles of boundary spanners, such as node 1 and node 4 in the three-dimensional example graph. Efficiency, as a closeness measure, attributed substantially centrality to the end users. Nevertheless, it does not distinguish the dependency from the influence. Therefore, a new index is needed in order to address these deficiencies. Bonacich's 'counting flow' has been widely recognized as a superior index among other conventional CNT-based measurements, to combine closeness and degree [36]. However, sociology's positive or negative impact is not applicable in studying coupled infrastructure behaviors, as dependencies and interdependencies in the interconnected system are hybrid in nature, e.g. directions and contents. In the new index, Efficiency-weighted Node Degree (EWND), the criticality of one node is associated with the criticalities of its neighboring edges, which are represented by their efficiencies. Models to correlate node degree with its adjacent edges' weights have been presented in social [37] and complex network studies [31] but they have the same problem as efficiency index, due to the fact that they cannot clarify dependency types. The EWND proposed here combines the ideas presented in [37] and [38], and utilizes the previously introduced complex-valued node degree for unidirectional graphs to explain orientation and categories of dependencies (the in-degree represents the dependency while the out-degree represents the importance). The adjacency matrices are used to calculate the EWND for each model. Using $h$ to represent an EPS node and $j$ to represent an ICT node, the equations to calculate the EWND for node $h$ and $j$ in the three-dimensional model are presented in (18) to (21). Similar equations for bidirectional and unidirectional graphs are not included here due to space limitations.

$$
\begin{aligned}
D_{h}^{i n} & =D_{e h}^{i n}+D_{c h}^{i n} \\
& =\sum_{y \in V_{e}, h \in V_{e}, y \neq h} a_{y h}^{e} \cdot \Delta E_{e, y h}+i \cdot \sum_{k \in V_{c}, h \in V_{e}, k \neq h} a_{j h}^{c} \cdot \Delta E_{c, j h}
\end{aligned}
$$

$$
\begin{aligned}
& D_{h}^{\text {out }}=D_{e h}^{\text {out }}+D_{c h}^{\text {out }} \\
& =\sum_{h \in V_{e}, y \in V_{e}, h \neq y} a_{h y}^{e} \cdot \Delta E_{e, h y}+\sum_{h \in V_{e}, k \in V_{c}, h \neq k} a_{h j}^{e} \cdot \Delta E_{e, h j} \\
& +i \cdot \sum_{h \in V_{e}, j \in V_{c}, h \neq j} a_{h j}^{c} \cdot \Delta E_{c, h j} \\
& D_{j}^{i n}=D_{e j}^{i n}+D_{c j}^{i n} \\
& =\sum_{y \in V_{e}, j \in V_{c}, y \neq j} a_{y j}^{e} \cdot \Delta E_{e, y j}+i \cdot\left(\sum_{y \in V_{e}, j \in V_{c}, y \neq j} a_{y j}^{c} \cdot \Delta E_{c, y j}\right. \\
& \left.+\sum_{l \in V_{c}, j \in V_{c}, l \neq j} a_{l j}^{c} \cdot \Delta E_{c, l j}\right) \\
& D_{j}^{o u t}=D_{e j}^{o u t}+D_{c j}^{\text {out }} \\
& =i \cdot\left(\sum_{j \in V_{c}, y \in V_{e}, j \neq y} a_{j y}^{c} \cdot \Delta E_{c, j y}+\sum_{j \in V_{c}, l \in V_{c}, j \neq l} a_{j l}^{c} \cdot \Delta E_{c, j l}\right)
\end{aligned}
$$

The results of efficiency-weighted node degree analysis for the example network in Fig. 3 are presented in Table IV. These results highlight node 2 and node 3's criticalities within the EPS and node 5 and 6's criticalities within the ICT system. All nodes play a dual role in both systems expect for node 1 .

TABLE IV EWND - Three-Layer Model Demonstration Graph

\begin{tabular}{|c|c|c|c|c|c|c|}
\hline Node & 1 & 2 & 3 & 4 & 5 & 6 \\
\hline$D^{\text {in }}$ & 0 & $0.8+0.4 \mathrm{i}$ & $0.7+0.3 \mathrm{i}$ & $0.4 \mathrm{i}$ & $0.5+1.4 \mathrm{i}$ & $0.7+1.2 \mathrm{i}$ \\
\hline$D^{\text {out }}$ & 0.5 & $0.9+0.5 \mathrm{i}$ & $0.7+0.7 \mathrm{i}$ & $0.4 \mathrm{i}$ & $0.4+1.2 \mathrm{i}$ & $0.3+0.8 \mathrm{i}$ \\
\hline
\end{tabular}

\section{H. Methodology Comparison}

The proposed indices display significance in the identification of interconnected system critical nodes/edges. Nevertheless, they also manifest inherent liability in the application of real system studies, which can be characterized into six aspects:

- Computational time: This aspect describes the computation complexity, the process requirement. A grade from 1 to 5, where 1 represents an expensive and ponderous performance and 5 represents a speedy process.

- Identification of critical nodes/edges: This aspect describes indices' effectiveness in identifying the group of most critical nodes/edges within the interconnected system, 1 for failure in identification of critical nodes and 5 for accurate identification of critical nodes.

- Identification of intraconnection nodes/edges: This aspect describes indices' effectiveness of the identification of the node or edge that bridges the two sub-clusters within its own system, 1 if the index cannot identify any bridging nodes/edges and 5 if the index can identify all of them.

- Identification of interconnection nodes/edges: It evaluates the successfulness of indices' identification of the node or edge that bridges the two sub-clusters between its own system and the other system. This aspect gives a grade of 1 if the index cannot identify any external bridging nodes/edges and 5 if it can identify all of them.

- Differentiation of marginal nodes: It assesses the ability of an index in differentiating the roles of boundary spanners (end users of the interconnected system). This 
aspect gives a grade of 1 if it cannot differentiate the marginal nodes from central nodes and 5 if it can accurately quantify the criticalities of marginal nodes.

- Differentiation of importance and dependency: This aspect rates the capability of an index in the differentiation of the importance and dependency a node has on its own and on the other system. A grade of 1 is given if the index cannot differentiate importance from dependency at all and 5 is given on the basis that the index can not only differentiate importance from dependency but can also quantify it accurately.

Based on the six aspects, a comparative diagram is plotted as shown in Fig. 5.

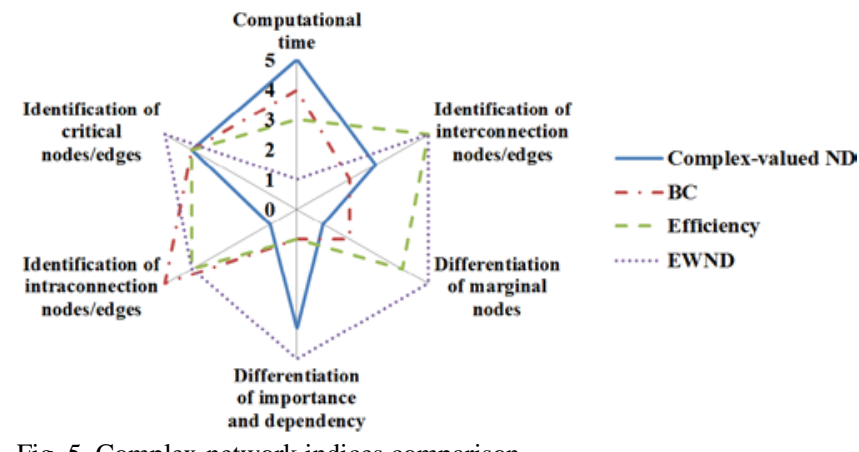

Fig. 5. Complex-network indices comparison

As shown in Fig. 5, Complex-valued Node Degree is most computationally efficient but less accurate in identifying interand intra- connected important nodes and marginal nodes in comparison to the other three standard indices. It provides though the information about 'highly-connected nodes' [2730] and the overall dependency structure of the CPS as shown in Fig. 11-14 which other indices cannot do. Similarly, Betweenness Centrality is most effective in identifying intraconnection nodes/edges and therefore it is most applicable in standalone system studies. It is slightly worse than the others though in differentiation of importance and dependency and differentiation of marginal nodes. Finally, the Efficiency is a very good index for identification of interconnection nodes/edges in a coupled systems study though not as good as Complex-valued Node Degree in differentiation of importance and dependency. It can be also seen that the newly proposed index, Efficiency-weighted Node Degree, overcomes the individual drawbacks of the other three standard indices and it is particularly good in identification of critical nodes and differentiation of importance from dependency. Because of this, it could be used to develop a look-up table for system operators to rank the most important hubs and to identify their vulnerability. The only drawback of this index is that it is more computationally demanding than the other indices. However, for the off-line studies, as the ones under consideration here, this is of very low importance. Finally, it is important to note, that due to the complexity of the problem under consideration, as in many other studies, a single index however versatile it might be, may not be an optimal solution and an engineering judgement must be used after considering information provided by several different indices.

\section{APPLICATION: VULNERABILITY ANALYSIS}

\section{A. Test System}

The test system, shown in Fig. 6, used to illustrate the concepts introduced in this paper is broadly based on interconnected distribution electrical power system (EPS) ICT network presented in [16]. It is a synthetic micro-grid network built during SINARI project and was previously used in [39-42]. It is suitably modified to incorporate different RES and loads in power network and different communication technologies in ICT network.

The EPS consists of 14 buses, 17 lines, 7 distributed generators (Fuel Cells, Wind Generators, Photovoltaic Generators modelled using corresponding daily production curves), 9 loads (domestic, commercial and industrial modelled using corresponding daily loading curves), and 3 HV/MV transformers. The electricity generation and consumption vary with time during the year, thus creating a time-dependent power flow for the electric part of the interconnected system. Depending on the loading, wind conditions and solar irradiation during the year, power flows in the network change and the distribution network could both import and export electric power from/to the external grid. Three specific timings of the year, $6470^{\text {th }}$ hour, $3582^{\text {nd }}$ hour, and $6559^{\text {th }}$ hour, representing the timings of annual maximum loading, minimum loading and average loading are selected to allow subsequent system analyses to be conducted.

The supporting ICT system includes 3 routers and 5 multiplexers as the information repeaters/aggregators and distributed information processing centers. The information channels connecting ICT components, utilize several communication technologies, namely, LAN-Giga Ethernet, WiMax, Ethernet and Optical Fiber, each with a corresponding bandwidth of $40 \mathrm{Gbps}, 75 \mathrm{Mbps}, 100 \mathrm{Mbps}$ and 10Gbps respectively [43-46]. The ICT link 2-23, uses Power over Ethernet (PoE) technology, for exchanging information between power bus 2 and central router 23, as well as supplying the power for the central router 23 from bus 2. The ICT network functions as a monitoring and control system of EPS, similar to a SCADA system. The state estimation data are collected at each electric bus and then forwarded to the corresponding communication hub. Data are aggregated and analyzed at the ICT site, facilitating a control command to be generated and issued to the EPS site. The information technologies are equipped with a technology-defined upload and download speed, allowing a sufficient amount of capacity to support the power distribution network. To constantly check the operating conditions of each power bus, the state estimation data are sent to the information aggregators periodically.

Note: It is important to highlight that different topologies of the ICT network (e.g., radial, as in this example, looped, star), the EPS network and the way how the two networks are interconnected would lead to different numerical results of the analysis presented in the following sections. The purpose of this study is to demonstrate the method and tools to analyse the interconnected electrical power and ICT networks and for that purpose the architecture of the two networks is not of a 
paramount importance as they are used for the illustration of the methodology only. The ICT network used in this study does not include rings or loops between any two nodes.

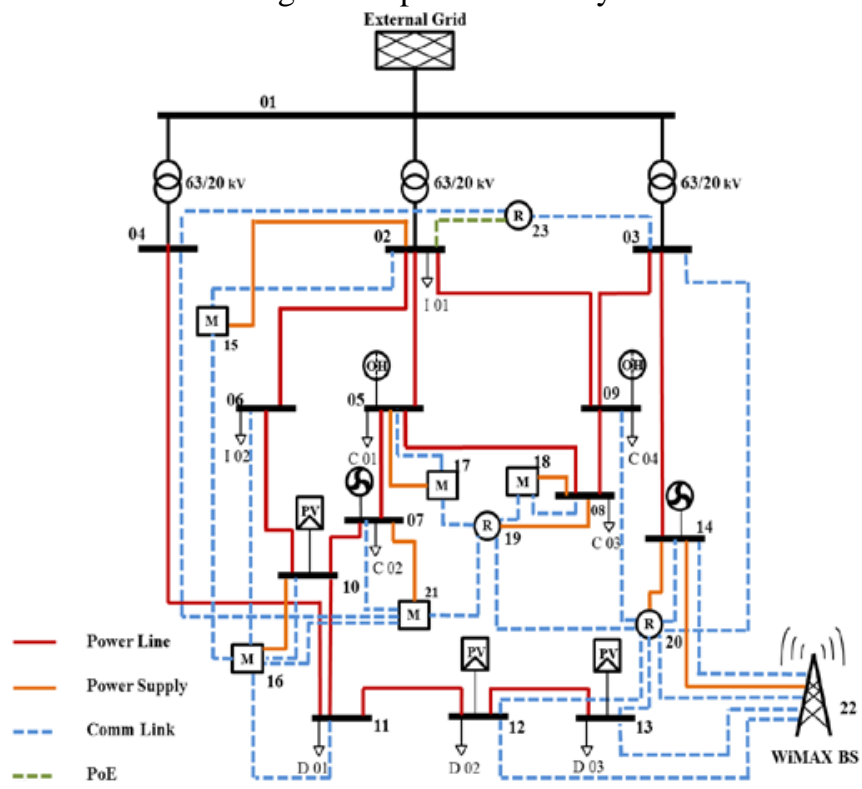

Fig. 6. 14-bus test system [16]

In the following subsections, a bidirectional graph describing the topology of a test power distribution network with supplementary ICT system is firstly presented. Following that, the directions of power and information under maximum, minimum and average loading conditions during the year are modelled in unidirectional models. Finally, the threedimensional model is introduced, which maintains the intrinsic properties of power system and communication system. It retains the integrity of the coupled infrastructure to the utmost extent and highlights the interactions between two systems. To rank the criticalities of each component, i.e. power buses, power lines, ICT routers/multiplexers, and the information channels, the coupled system is analyzed using Node Degree, Betweenness Centrality, Efficiency, and Efficiency-weighted Node Degree, as defined in Section II.

\section{B. Complex-network Modeling}

1) Bidirectional Graph: The test system is modelled as a graph where nodes represent power system's buses and communication routers/multiplexers, as shown in Fig. 7. Edges represent power lines and communication paths. Red nodes are the electric buses and pale blue nodes the routers/multiplexers. Bidirectional connections between two electric nodes are power flows between two buses (red solid lines), while edges between two ICT nodes are information exchange between two routers/multiplexers (pale blue dash dot lines). Real-time measurements from a power bus to the control center, or controlling command sent from a control center to a power bus, or power supply from a power bus to the communication network routers and multiplexers are modelled as an interconnection edge (dark green dash lines) between an EPS node and an ICT node. The bidirectional edges between any pair of nodes only represent the connectedness introduced by physical connection(s) between two nodes.

Fig. 7. Bidirectional model - test system

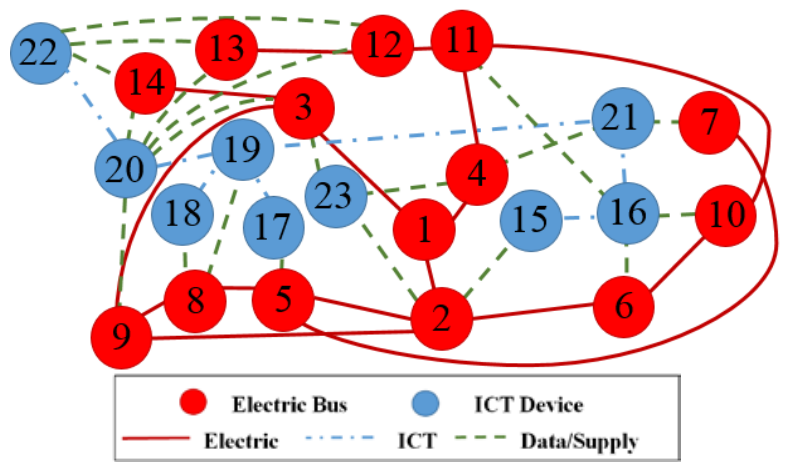

2) Unidirectional Graph: As shown in Fig. 8, the test system is modelled as a unidirectional graph where the green solid edges represent electricity supply to ICT nodes, and the light black dashed edges represent the control signal sent to electric buses. As explained previously, due to the variation of power consumption and generation, the power flow through a specific line might alter its direction. Therefore, the coupled network needs to be represented by different graphs according to different power flow directions.

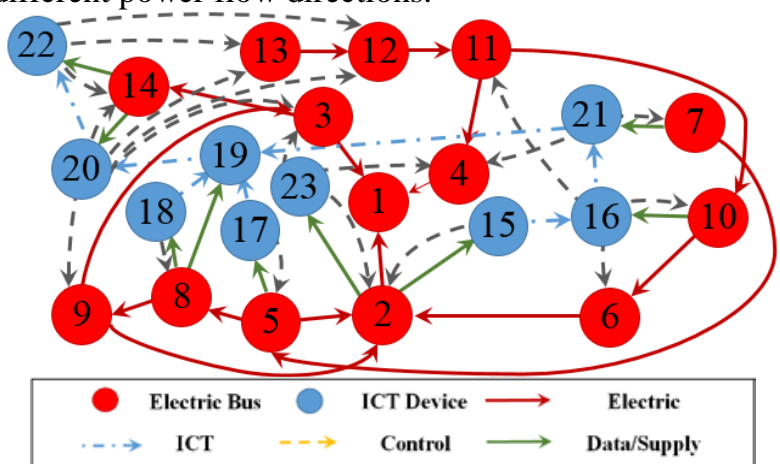

Fig. 8. Unidirectional model - test system (EPS maximum loading condition)

3) Three-dimensional Interconnected Model: Fig. 9 shows the three-dimensional model of test system. Power flows within the power system and the information exchange within the ICT system are modelled as unidirectional edges and bidirectional edges respectively, according to their real network nature as suggested in [16]. While in between these two layers, the unidirectional edges from EPS layer to ICT layer represent the power supplies, and the bidirectional edges between an EPS node and an ICT node represent the state estimation data from the EPS to the ICT system, and the command generated from the ICT system to the EPS.

\section{Vulnerability Analysis}

1) Complex-valued adjacency matrix: Fig. 10 presents the complex-valued adjacency matrix for test network bidirectional model. It displays a symmetry pattern in both EPS and ICT network. The upper left section presents the distribution of electric connections within the power network. The lower right section describes the internal cyber connections within the communication system. The upper right and lower left presents the interconnection edges, which can be either power supply to ICT system or the monitoring or control data flowing between EPS and ICT system. The adjacency matrix is an effective tool to reveal the 
interdependency categories and the distribution of connections and it facilitates the assessment of network properties such as Node degree, Betweenness Centrality and Efficiency.

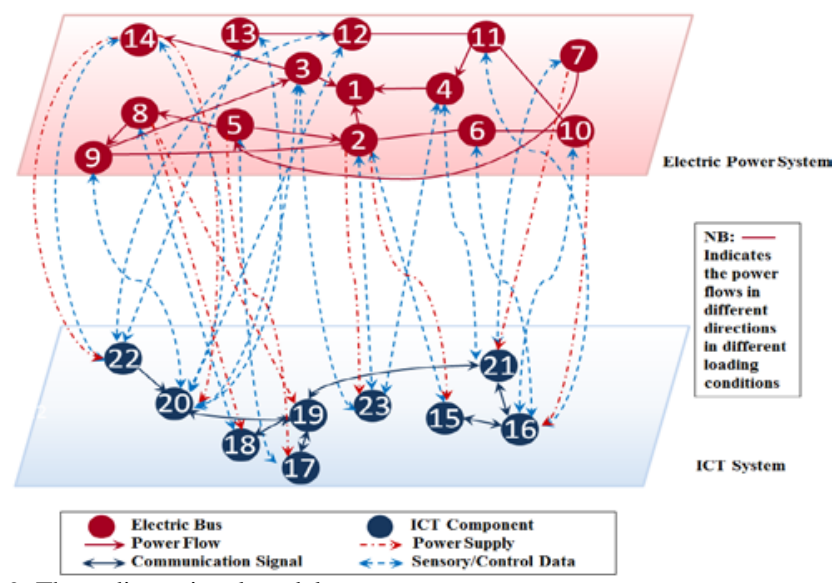

Fig. 9. Three-dimensional model - test system

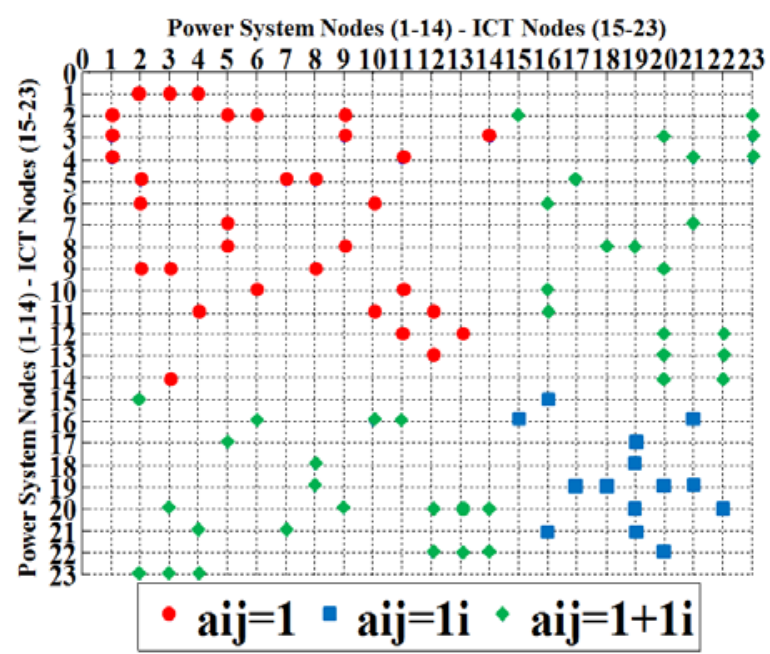

Fig. 10. Adjacency matrix - test system bidirectional model

2) Complex-valued node degree: The ND analysis for bidirectional graph highlights the importance of node 2 for power system, and node 20 for both EPS and ICT networks as it functions as the information processing center of the communication network and it is well connected with both networks.

The multi-sector frequency distributions of complex-valued ND for the bidirectional model, the unidirectional model and the three-dimensional model of the test network in power system maximum loading condition are presented in Fig. 11 14. The 3D histogram presented in Fig. 11 shows that all the nodes tend to have few ICT connections except for the central router such as 19 (corresponds to the peak $\mathrm{k}_{\mathrm{e}}=1$ and $\mathrm{k}_{\mathrm{c}}=5$ ) and 20 (corresponds to the peak $\mathrm{k}_{\mathrm{e}}=5$ and $\mathrm{k}_{\mathrm{c}}=7$ ) which either centrally process the information or issue the command. This is due to the topology of EPS network and the locallysupported ICT system. Compared with the ND analysis based on bidirectional graph, node's criticality is studied more specifically for each system in a unidirectional graph. Fig. 12 shows that there are no critical nodes for both systems. Interconnected nodes with one out-flow connection to power system tend to have at least one another connection to ICT system. Fig. 13 and Fig. 14 present the degree distribution for electric system and ICT network respectively. They highlight the interconnected nodes, since the entries of the interconnection edges are represented as $1+\mathrm{i}$, and the bidirectional ICT edges present each interconnected node with an in-degree and an out-degree. Fig. 13 and Fig. 14 also show that there are critical hubs within both systems, which are highly dependent on both systems. Node 20 in particular is identified as highly critical for the ICT system.

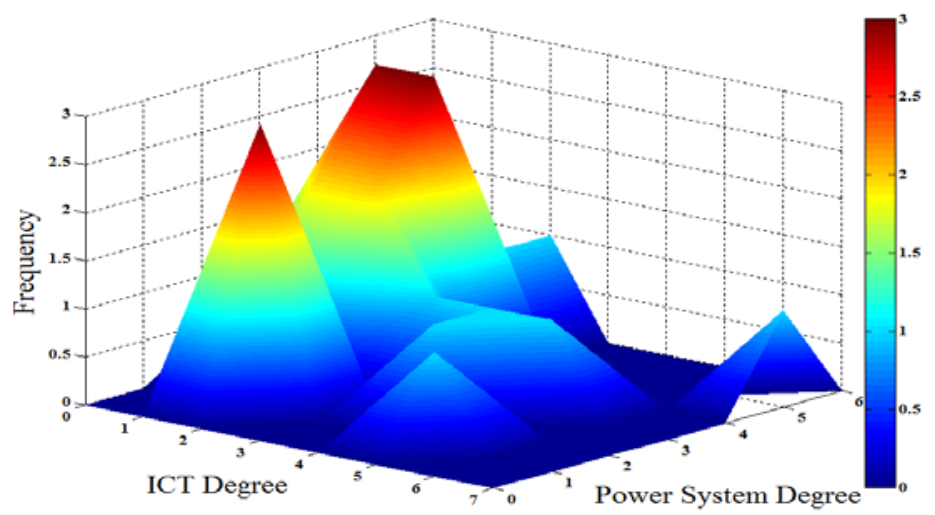

Fig. 11. Multiple infrastructure degree distribution - test system bidirectional model

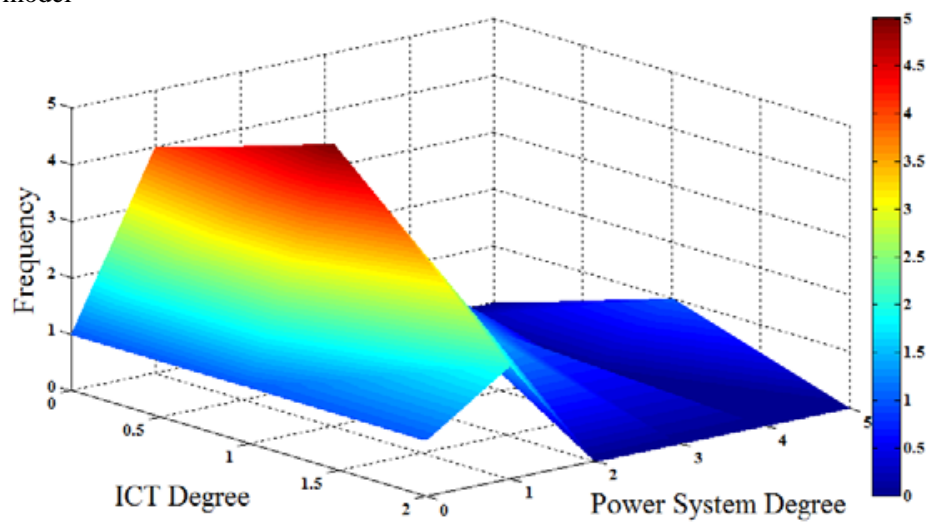

Fig. 12. Multiple infrastructure degree distribution - test system unidirectional graph out-degree (maximum loading condition)

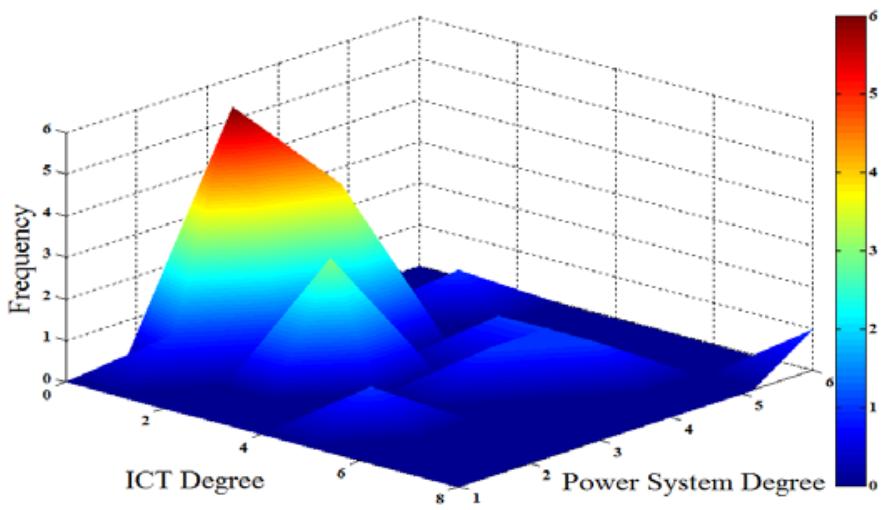

Fig. 13. Multiple infrastructure degree distribution - test system threedimensional model in-degree (maximum loading condition)

In conclusion, all three models identify the important EPS bus 2 and ICT router 20. The unidirectional graph and threedimensional model clarify the interdependency types in terms 
of direction, while three-dimensional model additionally highlights the importance of interconnected nodes such as EPS bus 14 and ICT hub 16.

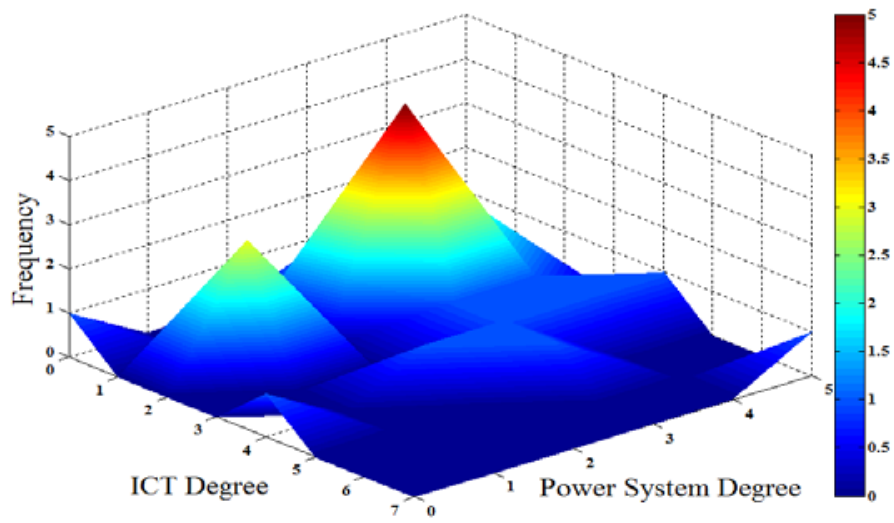

Fig. 14. Multiple infrastructure degree distribution - test system threedimensional model out-degree (maximum loading condition)

3) Betweenness Centrality (BC): The calculated node Betweenes Centralities for the three types of models of the test network are compared in Fig. 15. Nodes' betweenness centralities for bidirectional graph identify electric nodes 2 and 9 and ICT nodes 20 and 21 to be important for EPS and ICT network, respectively. The criticality of central router 19 , is revealed for the first time, which highlights the importance of using bidirectional graph to analyze ICT network. The graph also identifies a group of nodes which play a dual role in both infrastructures, including electric nodes 2, 3, 4 and ICT nodes $16,20,21,22,23$. The analysis of unidirectional graph and three-dimensional model under maximum loading condition identifies, as same as ND analysis, EPS bus 2 as the most important in EPS infrastructure. The interconnected ICT multiplexer 16 is identified as the most critical hub within the ICT system. Similar to the results from bidirectional graph, three-dimensional model highlights the importance of ICT central router 19. Furthermore, ICT node 18 is identified as one of the end-users for both infrastructures using bidirectional and unidirectional graphs, while threedimensional model identifies its role within ICT system (see Fig. 15). The difference is caused fundamentally by the fact that the bidirectional graph and unidirectional graph do not represent the dependencies introduced by $18-8$ and 19-8 based on their real networks' functionalities. Moreover, threedimensional model identifies the dual role players (e.g. node 2, 3 and 4) within the power system, as it has a two-way interactive impact on ICT system in terms of data transmission, while ICT system acts as the power receiver only. In both unidirectional graph and three-dimensional model, loading condition has little influence on the topological position of ICT nodes, due to the fixed topology and directions of data transmission of the ICT network. For the power system, however, different loading conditions could significantly increase or reduce EPS buses' centralities (e.g., node 11 plays a prominent role in power system under maximum loading condition, while under minimum and average loading, power flows from node 11 to node 13 , making node 13 an end-user of the power system). The effect of the change of loading condition is not demonstrated in figures due to space limitation but it is further discussed in efficiency analysis in the next section (see Fig. 17).

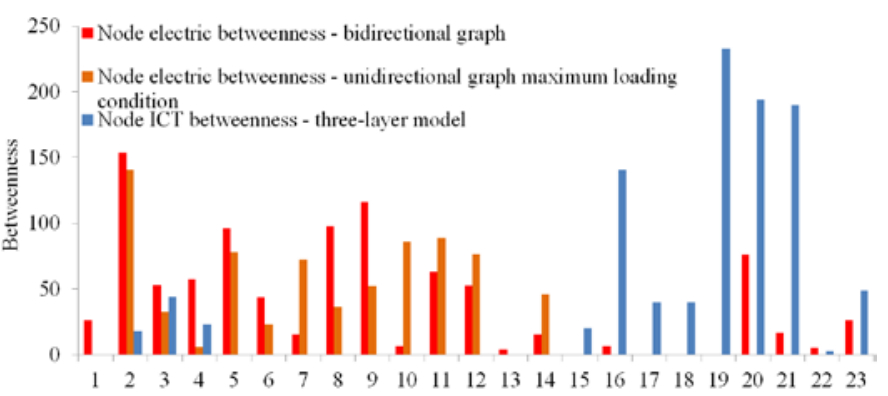

Fig. 15. Node betweenness centrality - test system

The edge betweenness has its significance in studying important interconnection edges. The centrality for bidirectional graph is presented in Fig. 16. Its symmetrical patterns can be found in the bidirectional graph adjacency matrix. It highlights the criticalities of edges 19-20 and 19-21 within the ICT network due to the high centrality of nodes 19 , 20 and 21. For the results obtained for unidirectional graph (not presented in figure), the edge centrality results highlight the importance of cyber link 16-21, power lines 12-11, 11-10, and power supply link 2-15 under maximum loading condition. It is also found that different loading conditions change the centrality of power lines (not presented in figure), e.g., line 11-12. The edge betweenness centrality for electric layer in the three-dimensional model, similar to the unidirectional case, illustrates the importance of power line 12-11 and 11-10. Furthermore, the three-dimensional model highlights the criticality of power line 8-9 in the power distribution network and edges $19-20$ and $19-21$ as the most critical ICT edges.

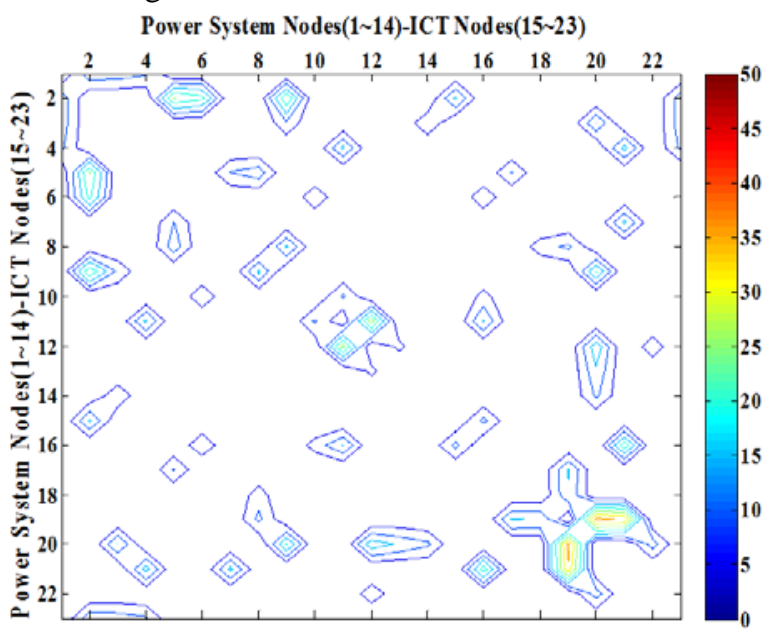

Fig. 16. Edge betweenness centrality - test system bidirectional model

In summary, betweenness centrality analysis highlights the criticality of EPS bus 9 and ICT central router 19 which has not been detected using previous methods and it demonstrates that different loading conditions affect the criticalities of nodes/edges. The results obtained using unidirectional electric layer of the three-dimensional model show consistency with the results from unidirectional graph while the results from 
ICT-layer are in agreement with the results from bidirectional graph. It is therefore essential to assess betweenness centrality for interconnected systems and to include directions of power/information flow in the model.

4)Efficiency: The node efficiency results from the electric part of unidirectional and three layer model (Fig. 17) identify electric nodes 2, 5 and 8 to be the most critical buses in EPS. In terms of the reliability of power supply, ICT node 15 and 23, are potentially vulnerable as a failure initiated within power system could easily pass down through critical power bus 2 which will result in the cut off of the power supply links 2-15 and 2-23. Moreover, ICT node 20 with 5 cyber connections to the EPS, plays the most important role in the controlling of EPS network, and nodes 19 and 16 are the second and the third most critical ICT components. The results also illustrate that the efficiency changes with power system's operating conditions (e.g. bus 10 and bus 14). In spite of the change though, the EPS buses 2, 5 and 8 remain the most critical buses, albeit with variations in their criticalities.

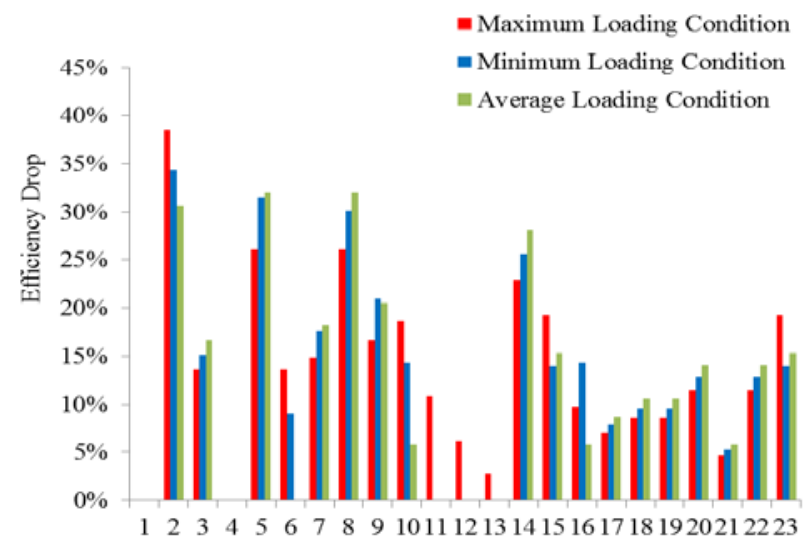

Fig. 17. Node electric efficiency - test system unidirectional model and threedimensional model under different power system loading conditions

Electric edge efficiency results for bidirectional graph (not presented in figure) highlight the importance of interconnection edges 2-15, 5-17, 8-18, and 8-19 for EPS, and edges $17-19,19-20,16-21$ and $19-21$ for the ICT network. These results highlight the importance of edges connected with critical nodes, as identified in node efficiency analysis.

Similarly, with the variation of loading conditions, the criticality of each power line changes. The results for ICT edge efficiency for unidirectional graph identifies edges 1920 and 21-19 as the most critical edges, while threedimensional model highlights the importance of edges 17-19 and 18-19, which is coherent with the results obtained from bidirectional graph. In the power network, power line 8-9 is highlighted for its central topological position. Power supply lines 2-15 and 2-23 with the same criticalities, are identified as the most vulnerable power supply links. However, as identified in the BC studies, edges 19-20 and 21-19 are in a more centralized position, either of which connects two cluster of the system as shown in Fig. 18. The difference in conclusions drawn from BC and Efficiency analysis are caused by the fact that betweenness values the importance based on topological connectedness while efficiency analysis takes into account distance, as given in (14). For instance, because node 22 is a 'distant' node to other nodes, links 17-19 and 18-19 are more centralized than 19-20 in spite of the fact that nodes 20 and 22 have higher degrees than nodes 18 and 19. To increase the efficiency of the network, therefore, the topological position of central routers needs to be considered carefully.

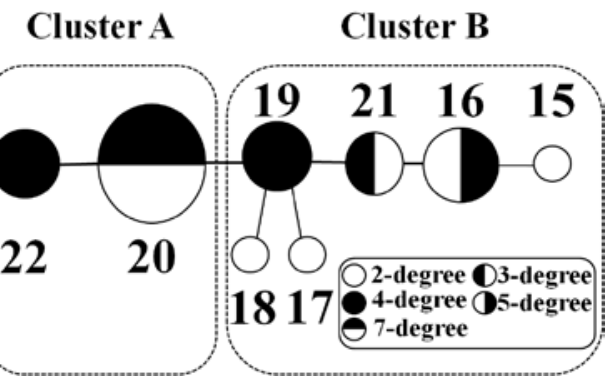

Fig. 18. Illustrative graph for ICT edges efficiency v.s. betweenness centrality

5) Efficiency-weighted node degree (EWND): The EWND results, presented in Figure 19, identify electric node 2 as the most critical node within the EPS, and nodes 16, 19 and 20 as the most critical ICT components. This is consistent with the results obtained from BC analysis. The EWND also suggests that the normal function of the multiplexer 16, the most important ICT hub for both infrastructures, highly depends on the correct operation of both systems. Particularly, central router 19, is reliably supplied with electricity. Although it is not directly controlling any electric buses, it takes the most critical role within the cyber network, which is in accordance with a typical star topology based ICT network.

The criticalities of ICT components are generally more easily identified using bidirectional graph, therefore there are more critical ICT hubs than EPS nodes. EWND provided an efficient tool to bridge the gap between topological centrality and connectedness, proving itself to be effective in multiinfrastructure studies.

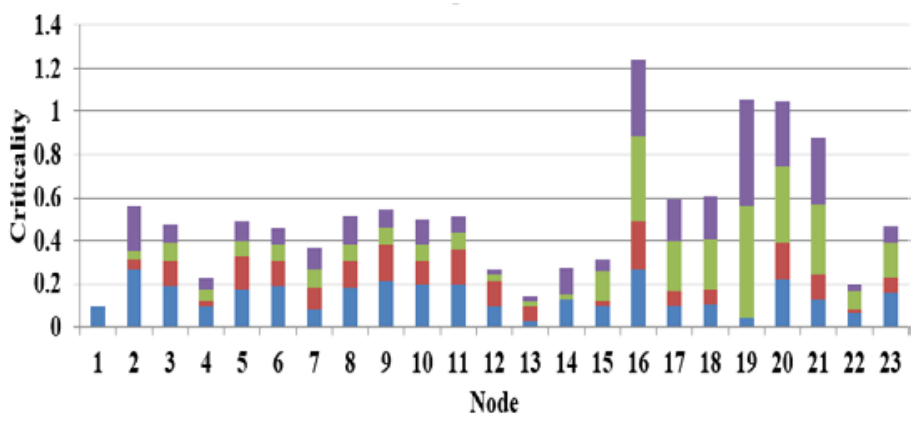

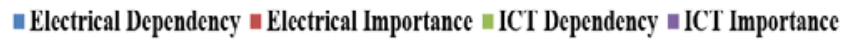
Fig. 19. Node global criticality - test system three-dimensional model (power system maximum loading condition)

\section{CONCLUSION}

This paper proposes a three-dimensional model to study the interdependencies and interactions of the interconnected heterogeneous infrastructure systems (cyber-physical network), based on Complex Network Theory. A bidirectional model and a unidirectional model are introduced in comparison with the three-dimensional model. It is shown from the results that the three-dimensional model, which 
integrates different topological patterns, is more accurate while flexible in modeling interconnected systems with different system behaviors. In addition, the three-dimensional model also shows its effectiveness in capturing different engineering structures (e.g., the change of power flow directions due to the change of loading conditions and different contributions from RES), which enables the criticality variation analysis of system components. It can be concluded from four different topological analyses that all four proposed graphical indices (ND, BC, Efficiency and EWND) show consistence in identifying the group of most critical electric and ICT nodes, with slight difference in the identification of the most critical ICT node. Among all topological indices, it is shown that the Efficiency and EWND analysis are most effective in identifying critical interconnected components. It is to be noted that, EWND, integrates Efficiency and Node Degree, bridging the gap between degree and position importance, allows the importance, as well as dependency of the interconnected system nodes to be categorized and quantified.

The main limitation of the proposed approach is that it is purely based on static topological structure of the interconnected network, in the sense that functional level modeling is not taken into account. Nevertheless, it provides a holistic modeling framework to study interconnected infrastructure systems, and with the integration of supplementary methods, the said limitation can be solved. Furthermore, the control theory can be developed based on the global modeling of CPS, which deserves more research efforts.

\section{REFERENCES}

[1] A.-L. Barabási and R. Albert, "Emergence of Scaling in Random Networks," Science, vol. 286, pp. 509-512, 1999.

[2] US-Canada power outage task force, "Final report on the august 14, 2003 blackout in the United States and Canada," Washington, WA, Apr 2004.

[3] ICS-CERT, "ICS-CERT Monitor ", U.S. Department of Homeland Security. Available Online: https://ics-cert.us-cert.gov/sites /default/files/Monitors/ICS-CERT\%20Monitor_Nov-

Dec2015_S508C.pdf

[4] E.F.Halpin, Cyberwar, netwar and the revolution in military affairs. Basingstoke England; Palgrave Macmillan, 2006.

[5] N. Falliere, L. O. Murchu, and E. Chien, "W32.stuxnet dossier," Feb 2011.

[6] S. A. Rinaldi, J. P. Peerenboom, and T. K. Kelly, "Identifying, understanding, and analyzing critical infrastructure interdependencies," IEEE Control Systems Magazine, vol. 21, pp. 11-25, Dec 2001.

[7] UCTE, "Final Report: System disturbance on 4 November 2006," Brussels, Belgium, 2007.

[8] S. De Porcellinis, R. Setola, S. Panzieri, and G. Ulivi, "Simulation of heterogeneous and interdependent critical infrastructures," International Journal of Critical Infrastructures, vol. 4, pp. 110-128, 2008.

[9] J. Johansson and H. Hassel, "An approach for modelling interdependent infrastructures in the context of vulnerability analysis," Reliability Engineering \& System Safety, vol. 95, pp. 1335-1344, 2010.

[10] B. Rozel, R. Caire, N. Hadjsaid, J. P. Rognon, and C. Tranchita, "Complex Network Theory and Graph Partitioning: Application to large interconnected networks," 2009 IEEE Bucharest Powertech, Vols 1-5, pp. 1673-1678, 2009.

[11] S. C. Müller, H. Georg, C. Rehtanz, and C. Wietfeld, "Hybrid simulation of power systems and ICT for real-time applications," in 2012 3rd IEEE PES ISGT Europe, 2012, pp. 1-7.
[12] A. Stefanov and C. C. Liu, "ICT modeling for integrated simulation of cyber-physical power systems," in 2012 3rd IEEE PES ISGT Europe, 2012, pp. 1-8.

[13] E. Zio and G. Sansavini, "Modeling Interdependent Network Systems for Identifying Cascade-Safe Operating Margins," IEEE Transactions on Reliability, vol. 60, pp. 94-101, 2011.

[14] D. W. Benbow and H. W. Broome, The certified reliability engineer handbook. Milwaukee, Wis.: ASQ Quality Press, 2009.

[15] Intelligent monitoring, control, and security of critical infrastructure systems. New York: Springer, 2014.

[16] J. L. S.Torres, "Vulnerability, interdependencies and risk analysis of coupled infrastructures: power distribution network and ICT," Ph.D. dissertation, Dept. Electric Power Eng., Univ. Grenoble, Alpes, France, 2013.

[17] S. Boccaletti, V. Latora, Y. Moreno, M. Chavez, and D. U. Hwang, "Complex networks: Structure and dynamics," Physics Reports-Review Section of Physics Letters, vol. 424, pp. 175-308, Feb 2006.

[18] J. Wei and D. Kundur, "Biologically Inspired Hierarchical CyberPhysical Multi-agent Distributed Control Framework for Sustainable Smart Grids," in Cyber Physical Systems Approach to Smart Electric Power Grid, K. S. Khaitan, D. J. McCalley, and C. C. Liu, Eds., ed Berlin, Heidelberg: Springer Berlin Heidelberg, 2015, pp. 219-259.

[19] V. Rosato, L. Issacharoff, F. Tiriticco, S. Meloni, S. De Porcellinis, and R. Setola, "Modelling interdependent infrastructures using interacting dynamical models," Int. J. Critical Infrastructures, vol. 4, pp. 63-79, 2008.

[20] L. C. Freeman, "Centrality in Social Networks Conceptual Clarification," Social Networks, vol. 1, pp. 215-239, 1979.

[21] S. L. Wang, L. Hong, and X. G. Chen, "Vulnerability analysis of interdependent infrastructure systems: A methodological framework," Physica a-Statistical Mechanics and Its Applications, vol. 391, pp. 3323-3335, June 12012.

[22] S. Boccaletti, V. Latora, Y. Moreno, M. Chavez, and D. U. Hwang, "Complex networks: Structure and dynamics," Physics Reports-Review Section of Physics Letters, vol. 424, pp. 175-308, Feb 2006.

[23] M. Parandehgheibi and E. Modiano, "Robustness of Interdependent Networks: The case of communication networks and the power grid," 2013 IEEE Global Communications Conference (Globecom), pp. 21642169, 2013.

[24] X. M. Liu, H. E. Stanley, and J. X. Gao, "Breakdown of interdependent directed networks," Proceedings of the National Academy of Sciences of the United States of America, vol. 113, pp. 1138-1143, Feb 22016.

[25] P. Pederson, D. Dudenhoeffer, S. Hartley, and M. Permann, "Critical infrastructure interdependency modeling: a survey of US and international research," Idaho National Laboratory, Idaho Falls, 2006.

[26] E. Estrada, The structure of complex networks : Theory and applications. Oxford, U.K.: Oxford University Press, 2012.

[27] D. S. Callaway, M. E. J. Newman, S. H. Strogatz, and D. J. Watts, "Network robustness and fragility: Percolation on random graphs," Physical Review Letters, vol. 85, pp. 5468-5471, Dec 182000.

[28] Z. Wang, A. Scaglione, and R. J. Thomas, "The Node Degree Distribution in Power Grid and Its Topology Robustness under Random and Selective Node Removals”, 2010 IEEE International Conference on Communications Workshops, 2010, pp. 1-5.

[29] R. Albet, N. Jeong, and A. L. Barabasi, "Error and attack tolerance of complex networks (vol 406, pg 378, 2000)," Nature, vol. 409, pp. 542, Jan 252001.

[30] S. V. Buldyrev, R. Parshani, G. Paul, H. E. Stanley, and S. Havlin, "Catastrophic cascade of failures in interdependent networks," Nature, vol. 464, pp. 1025-1028, 2010.

[31] T. H. Cormen, C. Stein, R.L.Rivest, and C. E. Leiserson, Introduction to algorithms: Cambridge: MIT Press, 2009.

[32] L. C. Freeman, "A set of measures of centrality based on betweenness," Sociometry, vol. 40, Mar 1977.

[33] R. Kinney, P. Crucitti, R. Albert, and V. Latora, "Modeling cascading failures in the North American power grid," European Physical Journal B, vol. 46, pp. 101-107, Jul 2005.

[34] V. Latora and M. Marchiori, "Efficient behavior of small-world networks," Physical Review Letters, vol. 87, Nov 52001.

[35] V. Rosato, S. Bologna, and F. Tiriticco, "Topological properties of high-voltage electrical transmission networks," Electric Power Systems Research, vol. 77, pp. 99-105, Feb 2007.

[36] J. M. Bolland, "Sorting out centrality: An analysis of the performance of four centrality models in real and simulated networks," Social Networks, vol. 10, pp. 233-253, 1988. 
[37] T. Opsahl, F. Agneessens, and J. Skvoretz, "Node centrality in weighted networks: Generalizing degree and shortest paths," Social Networks, vol. 32, pp. 245-251, Jul 2010.

[38] P. Crucitti, V. Latora, and M. Marchiori, "Model for cascading failures in complex networks," Physical Review E, vol. 69, Apr 2004.

[39] J. Mcdonald, H. Decroix, R. Caire, J. Sanchez, S. Chollet, N. Oualha, A. Puccetti, A. Hecker, C. Chaudet, H. Piat, D. Georges, and F. Planchon, "The SINARI project: Security analysis and risk assessment applied to the electrical distribution network," in Electricity Distribution (CIRED 2013), 22nd International Conference and Exhibition on, 2013, pp. 1-4.

[40] J. Sanchez, R. Caire, and N. Hadjsaid, "Application of Hermitian Adjacency Matrices for coupled infrastructures interdependencies analysis," in IEEE PES ISGT Europe 2013, 2013, pp. 1-5.

[41] B. Stahl, L. L. Thanh, R. Caire, and R. Gustavsson, "Experimenting with Infrastructures," in Critical Infrastructure (CRIS), 2010 5th International Conference on, 2010, pp. 1-7.

[42] R. Caire, S. J, Sanchez, and N. Hadjsaid, "Vulnerability analysis of coupled heterogeneous critical infrastructures: A Co-simulation approach with a testbed validation," in IEEE PES ISGT Europe 2013, 2013, pp. 1-5.

[43] S. Heuel and H. Mellein, "Mobile WiMAX throughput measurements application note ", Munich, Germany.

[44] IEEE standard for ethernet, Section 6, IEEE Std 802.3-2012, 2012.

[45] U. V. Burg, The triumph of Ethernet : technological communities and the battle for the LAN standard. Stanford, Calif.: Stanford University Press, 2001.

[46] S. Yao, "Polarization in fiber Systems: squeezing out more bandwidth," New York, NY, 2003.

Jovica V. Milanović (M'95, SM'98, F'10) received the Dipl.Ing. and M.Sc. degrees from the University of Belgrade, Belgrade, Yugoslavia, the Ph.D. degree from the University of Newcastle, Newcastle, Australia, and the Higher Doctorate (D.Sc. degree) from The University of Manchester, U.K., all in electrical engineering. Currently, he is a Professor of Electrical Power Engineering, Deputy Head of School and Director of External Affairs in the School of Electrical and Electronic Engineering at the University of Manchester, U.K., Visiting Professor at the University of Novi Sad, Serbia, University of Belgrade, Serbia and Conjoint Professor at the University of Newcastle, Australia. Professor Milanovic is a Chartered Engineer in the UK, Foreign member of the Serbian Academy of Engineering Sciences, Fellow of the IET, Fellow of the IEEE, Distinguished IEEE PES Lecturer and currently serves on IEEE PES Governing Board as Regional Representative for Europe, Middle East and Africa and on the IEEE PES Fellows Committee.

Wentao Zhu (S'16) received the B.Eng degree in electrical and electronic engineering in 2015 from the University of Manchester, Manchester, U.K., where he is currently pursuing the Ph.D. degree. 